\title{
GÉNESIS MENTAL DEL SUSTANTIVO
}

En el año 1974, en su presentación de la lingüística guillaumiana, Alicia Yllera pensaba que "incluso se podría considerar la única escuela francesa relativamente original y autónoma". Y, después de indicar que hasta un partidario de Hjelmslev como Togeby había aprovechado algunos de sus resultados, señalaba cierta influencia de Guillaume sobre algunos gramáticos o lingüistas españoles como Alarcos, Roca Pons, Hernández Alonso, etc., principalmente en el campo del verbo ${ }^{1}$.

De todas formas, la publicación, directamente en castellano, en el año 1975, de la tesis doctoral de Molho', pudo ayudar a los estudiosos españoles a descubrir o profundizar las opciones teóricas de la psicomecánica de Guillaume, y, en particular, su aplicación al caso de los aspectos, de los modos y de los tiempos del verbo castellano. Además, amén de sus primeras obras sobre el verbo y de una serie de artículos redactados de 1933 a 1958 y recogidos en un libro, ya han salido a la luz 5 volúmenes de las lecciones leídas en París por el maestro durante los años 1938-1960, a los que se puede aducir un compendio de sus concepciones pu-

1 “La lingüística guillaumiana”, $F M, 15$ (1974-75), pp. 58-59. Huelga decir que es también idea de los seguidores de Guillaume, y en particular de VALIN, su discípulo más ortodoxo, que en 1971 consideraba al estructuralismo guillaumiano como un estructuralismo "pas comme les autres"' (R. VALIN, "Introduction", en Gustave Guillaume, Leçons de linguistique 1948-49), t. 1, serie A, Structure semilogique et structure psychique de la langueifrançaise I, Presses de l'Université Laval, Québec, 1972; Klincksieck, Paris, 1971, p. 58; véase infra n. 6.

${ }_{2}$ Mauricio Molno, Sistemática del verbo español (aspectos, modos, tiempos), Gredos, Madrid, 1976, 2 ts. 
blicado en $1973^{3}$. Relativamente a la edición completa de sus papeletas manuscritas, cuya preparación necesitará por lo menos la colaboración de dos generaciones de discípulos ${ }^{4}$, parece aún muy poca cosa, pero esos libros facilitan ya una buena comprensión del guillaumismo.

\section{LA LINGÜÚSTICA GUILLAUMIANA ES UNA LINGÜÍSTICA OPERATIVA}

Como lo ha subrayado ya el lingüista búlgaro Mantchev, la psicomecánica merecería calificarse de lingüística operativa $a^{5}$. En efecto, la piedra angular de la teoría es como un postulado que se podría enunciar de esta forma: si se acepta que toda práctica del lenguaje implica una actividad mental concomitante, entonces hay que admitir también que, tributario de operaciones del pensamiento, venga acompañada de cierto transcurso de tiempo, aunque sea mínimo. A lo mejor algún día la neurolingüística conseguirá precisar el lugar del cerebro donde nace y se apaga nuestra actividad mental en sus distintas etapas, y logrará medir su duración cuando funciona. De momento, y a falta de toda prueba instrumental o experimental, la psicomecánica ${ }^{6}$ queda con-

${ }^{3}$ Gustave Guillaume, Temps et verbe. Théorie des aspects, des modes et des temps (1929), y L'architectonique du temps dans les langues classiques (1943), reimpr., Champion, Paris; Langage et science du langage, Presses de l'Université Laval, Québec; Nizet, Paris, 1964 (recopilación de 19 artículos aparecidos entre 1933 y 1959); Legons. . . (cit. n. 1): Leģons de linguistique 1948-49, t. 2, serie B, Psychosystématique du langage. Principes, méthodes et applications I, Presses de l'Université Laval, Québec; Klincksieck, Paris, 1971; Legons de linguistique 1948-49, t.3, serie C, Grammaire particulière du français et grammaire générale IV, Presses de l'Université Laval, Québec; Klincksieck, Paris, 1973; Principes de linguistique théorique (colección de textos inéditos preparados bajo la dirección de VALIN), Presses de l'Université Laval, Québec; Klincksieck, Paris, 1973; Leçons de linguistique 1949-50, t.4, serie A, Structure sémiologique et structure psychique de la langue française II, Presses de l'Université Laval, Québec; Klincksieck, Paris, 1974. La bibliografía más reciente de los trabajos psicomecánicos es la de LiONEL MENEy y Hervé CuRat, Bibliographie de la recherche en psycho-systématique du langage, 2 fasc., Université Laval, Québec, 1980-81.

${ }^{4}$ Valin, "Avant-propos", en Guillaume, Legons. . ., t. 4, p. 12.

${ }^{5} \mathrm{~K}$. MANTCHEV, La genèse de la phrase simple énonciative dans la structure générale de la langue française contemporaine, Presses de l'Université de Sofia, Sofia, 1976, p. 30 , n. 14 y pp. 115-116.

6 '"L'impossibilité dans l'état actuel de nos moyens de connaissance de mesurer le temps opératif par nous appelé glossologique, temps porteur des opérations instantanées dont fait état la psychomécanique du langage, n'est donc pas une objection à retenir contre elle. [ . . .]. Bien sûr, le jour où la con- 
vencida de que para pensar hace falta tiempo. A ese tiempo particular necesario para poner en obra el lenguaje, positivo aunque probablemente infinitesimal, se le ha dado el calificativo de operativo $^{7}$. Postulada su existencia, resulta posible asociar a

naissance des mécanismes neurophysiologiques sur lesquels reposent l'edification et le fonctionnement du langage aura suffisamment progressé et où la trace des opérations postulées aura été retrouvée, on pourra procéder à des mesures qui viendront apporter une confirmation de plus, et éclatante, á l'exactitude de la théorie, mais la théorie n'a aucunement besoin d'attendre une telle preuve pour être reconnue comme scientifique. [...]. C'est qu'une science du mentalisme linguistique doit nécessairement prendre son départ à des apparences mentales qui, bien qu'accesibles par la seule voie de l'instropection, constituent un premier ordre de réalité [...] et qu'elle doit ensuite non moins nécessairement, en se fondant sur les seuls moyens et ressources de l'imagination, accéder à une réalité mentale cachée d'un autre ordre, ordre second de réalité qui, précisément parce qu'il est caché et mental, échappe à toute introspection et dont, par conséquent, l'existence se dérobe à notre conscience" (VALIN, "Introduction", p. 37). Puede interesar también VaLin, "Des conditions d'existence d'une science du mentalisme", LMo, 62 (1968), 297-309; y L. M. SKreLina, "Le temps opératif et la structure de la phrase", en Langage et psychomécanique du langage (Hommage à $\mathrm{R}$. Valin), Presses de l'Univ. de Lille et de l'Univ. Laval, Lille-Québec, 1980, p. 87.

${ }^{7}$ Si la separación, dentro del lenguaje, de los dos planos de la potencia y del efecto aparece ya en Guillaume (Le problème de l'article et sa solution dans la langue française, Hachette, Paris, 1919, reed. Nizet, Paris; Presses de l'Université Laval, Québec, 1975), hay que esperar diez años y la publicación de Temps et verbe... para que aparezca el concepto de tiempo operativo, como se puede ver en las dos citas siguientes, entresacadas de estas dos obras, respectivamente: "Au moment de l'emploi, la notion permanente est appelée du fond de l'esprit pour paraître à la surface. Il lui faut ainsi traverser les diverses couches d'impression étagées au-dessus d'elle (p. 161); “La pensée en action de langage exige réellement du temps. Il y a là un principe de grande portée en linguistique psychologique et en linguistique générale"' (p. 8, n. 1). Sobre el particular, véase VALIN, “Introduction”, p. 14; Guillaume, Langage. . ., p. 109, y $L$ 'architectonique..., p. 17. Se pueden ver algunas reacciones - positivas o negativas - suscitadas por el postulado del tiempo operativo en J. B. CARroll (reseña de Valin, “Introduction”), Lan, 30 (1954), 586-591; H. ConTreras (reseña de VALIN, La méthode comparative en linguistique historique et en psychomécanique du langage, Presses de l'Université Laval, Québec, 1964), BHi, 68 (1966), 174-176; R. A. HAll (reseña de W. HIRTLE, The simple and progressive forms; an analytical approach, Presses de l'Université Laval, Québec, 1967), RPh, 23 (1969-70), 230-234; R. L. WAGNer, Grammaire et philologie, C.D.U., Paris, 1953 , p. 149; A. HENRY, “Linguistique et stylistique à propos de l'article français", $B A B, 48$ (1962), 312-322; K. Togeby (reseña de Guillaume, "La représentation du temps dans la langue française" y "De la double action séparative du present dans la représentation française du temps", ambos reimpresos en Langage..., pp. 184-219), 'Alin, 9 (1965), 107-109; K. HEGER (reseña de G. Molgnet, Essai sur le mode subjonctif en latin postclassique et en ancien français, P.U.F., Paris, 1959); ZRPh, 77 (1961), 148-158; M. CoHEN, BSLP, 
cada acto lingüístico solidario de una serie de operaciones mentales un eje de desarrollo en total coincidencia con el del transcurso de tiempo operativo que le sirve de soporte y de vehículo ${ }^{8}$. De ahí que se utilicen figuras que, evidentemente, no pretenden brindar la imagen fiel de una realidad todavía por descubrir, pero que sí pueden ayudar a percibir relaciones, asentar puntos de referencia estáticos, representar mecanismos dinámicos, y, finalmente, favorecer la ordenación de las distintas etapas de las operaciones mentales subyacentes ${ }^{9}$.

\section{PResentación}

Sin procurar presentar los cimientos de la psicomecánica ${ }^{10}$, quisiera ahora hacer tan sólo hincapié preferentemente en la representación del vocablo o parte de lengua a la que puede conducir para nuestros idiomas hermanos. Se trata efectivamente de un concepto básico en la medida en que, como se ha recalcado repetidas veces, y sobre todo en comparación con la lingüística de inspiración chomskyana, la psicomecánica queda ante todo como una lingüística de la palabra ${ }^{11}$. En su teoría, la palabra ocupa un si-

47 (1951), 2-5. Según Valin, las resistencias que encontró el concepto de tiempo operativo entre los lingüistas quizás hubieran sido menores si su inventor hubiera distinguido entre el tiempo discursivo y el tiempo glosológico, y si hubiera explicitado claramente las relaciones que unen estas dos especies de tiempo, encerradas en el tiempo praxeogenético, con el tiempo glosogenético y, a través de él, con el tiempo cósmico ("Introduction", p. 33).

${ }^{8}$ Marc Wilmet la evoca como "hypothèse majeure" (Gustave Guillaume et son école linguistique, Nathan, Paris; Labor, Bruxelles, 1972, p. 17).

9 “'Un jour viendra sans doute où, devenues insuffisantes, nos représentations d'ajourd'hui seront remplacées par une algèbre spéciale qui dégagera la pensée des chercheurs d'alors de ce que nos schémas actuels conservent de trop matériel; mais avant d'en arriver là, il reste à la psychomécanique un long chemin à parcourir et, selon toute vraisemblance, à épuiser les potentialités de la méthode actuelle" (Roch Valin, Petite introduction à la psychomécanique du langage, Presses de l'Université Laval, Québec, 1954, p. 25).

${ }^{10}$ En el ámbito hispánico, han sido presentados en español por A. YLLERA (art. cit., n. 1); M. Molho (op. cit., n. 2) y también por M. Joly, "Guillaume y la ciencia del lenguaje", $N R F H, 22$ (1973), 78-85.

11 " [...] la linguistique de G. Guillaume est fondamentalement une linguistique du vocable, du vocable en tant que forme, ou, pour nous limiter à ce que montre la typologie des langues indo-européenes, disons: une linguistique du mot [...]. Linguistique du mot et non, au moins au départ, linguistique de la phrase" (G. Morgnet, "Presentation de la psychomécanique du langage", en Grammaire générative et psychomécanique du langage, eds. S. de Vriendt, J. Dierickx y M. Wilmet, Didier, Paris; AIMAV, Bruxelles, 1975, p. 11). 
tio privilegiado como resultado de un primer momento de la edificación del lenguaje, y como punto de partida para la segunda etapa que produce el lenguaje efectivo. A la vez término y origen, fin y principio, sirven de umbral entre las dos fases por las que debe pasar toda la construcción del habla, entre la ausencia total de lenguaje y su realización y manifestación concretas, y encierra en sí todas sus posibilidades combinatorias en el marco de la oración -léxicas, gramaticales, fonéticas.

Pero antes de proponer una descripción de los estratos más relevantes que el análisis descubre en la constitución interna del vocablo románico, nos detendremos algunos instantes en la nueva explotación que hizo Guillaume de la consabida dicotomía saussuriana lengua / habla' ${ }^{12}$.

REINTERPRETACIÓN GUILLAUMIANA DE LA DICOTOMÍA LANGUE / PAROLE

\section{Doble relación del lenguaje con el tiempo}

La génesis del lenguaje.- Está claro que, en el lenguaje considerado globalmente, el tiempo, el tiempo cósmico está presente dos veces. Decir que cada idioma tiene su historia es decir que no deja de proseguir constantemente una elaboración cuyo pasado más remoto no llega siempre a conocer completamente la lingüística, ni tampoco logra predecir su futuro. Esa labor gradual interrumpida, aunque obviamente colectiva, procede realmente de las iniciativas individuales, por lo menos de las que, sometidas al veredicto de la comunidad, han sido recogidas e institucionali-

${ }^{12}$ La falta de una correspondencia total entre la dicotomía saussuriana y la guillaumiana ha sido destacada varias veces. Remitimos por ejemplo a lo que escribieron VALIN ("Aussi le lecteur est'il invité à ne pas identifier trop vite glosso[genèse] et diachronie ou praxéo [genèse] et synchronie, car s'il existe un rapport manifeste entre le concept guillaumien de glosso [genèse] et le concept saussurien de diachronie, il n'existe qu'une lointaine analogie entre ceux de praxéo [genèse] et de synchronie", "Introduction", p. 23: la terminología se aclara en el $\$ 1.1 \mathrm{~b}$ ); A. ÝlLerA (art. cit., pp. 68-70). Se pueden consultar también S. Clarke y R. Sheen, "Comments on Guillaume's langue / discours in the light of Chomsky's competence / performance dichotomy", en Grammaire générative transformationnelle et psychomécanique, eds. A. Joly et al., Éditions Universitaires, Paris, 1973, pp. 31-54; J. STEFAnini, "Sur la conception guillaumienne de l'opposition langue / discours", TLL, 11 (1973), 319-328; J. Hewson, "Langue and parole since Saussure", HistL, 3 (1976), 315-348; M. LaUNAY, "Langue, discours et pensée. Une lecture de la grammaire systématique", $M C V, 13$ (1977), 425-446. 
zadas, por enriquecedoras ${ }^{13}$. Lo que nos da como resultado es un producto acabado, duradero aunque inestable, totalmente inmaterial, a pesar de que propone e impone evidentemente unos patrones de materialización: algo completamente construido, aunque siempre prometido a mejoras, algo abstracto pero capacitado para concretarse en caso de necesidad, es decir en caso de utilización. Esa parte del lenguaje inaccesible por la observación directa, conocida desde Saussure como la lengua, se hereda, se lega, y evoluciona con el tiempo. Inestable por naturaleza, como hemos puntualizado, por función aparece duradera y permanente. $Y$ por dos razones: $1^{\circ}$ ) porque, en cada individuo de la comunidad idiomática, nunca deja de estar presente, si no su totalidad colectiva, por lo menos de lo que de ella ha sabido recoger y atesorar cada uno, lo que le basta para permitir un intercambio suficiente; $2^{\circ}$ ) porque, si cada persona a lo largo de su existencia - consciente - no cesa de ampliar y mejorar - a reserva de alteración física o de disturbio orgánico- su propia capacidad, esas modificaciones positivas, generalmente inconscientes, no amenazan verdaderamente su cohesión ni su coherencia internas ${ }^{14}$. Así

13 VALIN ha dedicado algunas páginas al problema de las condiciones en las que, dentro de la relación glosogénesis-praxeogénesis, aparecen las innovaciones lingüísticas ("Introduction", pp. 27-30 y 55-57). De sus sugerencias, recogeremos los dos puntos siguientes: $\mathbf{I}^{\circ}$ ) la innovación se relaciona naturalmente con la percepción - real o imaginaria - en lo que de la lengua se conoce en un momento dado de una carencia revelada por una necesidad expresiva considerada como nueva; $2^{\circ}$ ) cuando se da realmente la innovación, ésta procede de las posibilidades disponibles: ' $G$ 'est donc bien en praxéo[genèse] [...] qu'une innovation réussie se produit $[\ldots]$ une praxéo[genèse] qui appelle un dépassement $[. .$.$] et suppose, pour ce faire, un recours à la créativité inhéren-$ te à la mystérieuse faculté de langage aux fins d'obtenir une solution généralisée et permanente du problème posé, laquelle solution $[\ldots]$ constituera un apport à la glosso[genèse]" (ibid., p. 57).

14 " 'Ce qui fait la réalité de la glosso[genèse], c'est la persistance [...] des espaces imaginaires de configuration ou systèmes qui font la réalité formelle d'une langue, tous et chacun de ces systèmes, ainsi que l'ensemble systématique qu'ils constituent, étant vus exister conjointement et simultanément en chacun des instants du temps cosmique en progression" (ibid., p. 52). "[...] il existe, en glosso[genèse], une triple visée; a) uné visée que nous appellerons systématique tendant à la construction et à la continuation, par dépassement des systèmes constitutifs de la langue; b) une visée sémiologique, qui est recherche indéfiniment poursuivie d'une sémiologie suffisamment apte à extérioriser les articulations du système et, plus généralement, les contrastes de tous ordres que la pensée institue en elle même à des fins de concevabilité et qui, pour atteindre son but, profite de tous les accidents de la contingence historique; c) enfin une visée que nous appellerons pléronomique allant à une diversification croissante et à un accroissement indéfini du nombre des idées particuliè- 
pues, el tiempo cósmico, que nos propone una especie de imagen idealizada de la duración, sirve de soporte a una primera clase de actividad lingüística.

La génesis del discurso. - Por otro lado, bien se sabe que sirve también para otra actividad, la que consiste en valerse de la lengua, tal como se la posee - cuantitativa y cualitativamente-, a fin de elaborar secuencias de habla concreta, materializadas y apropiadas a un deseo particular de significar algo. Se trata aquí, en comparación con lo anterior, de un acto de explotación de la lengua, individual -o individualizado si se imagina el caso de varios hablantes que se expresan mediante la misma habla- y también de un acto momentáneo y discontinuo, condenado a ser efímero en su realidad fónica antes de la invención del magnetófono. En vez de un producto único perdurable aunque inestable, recogemos aquí una multitud de productos, indefinidamente distintos por su cantidad y sustancia propias: las oraciones, cuyo papel singular - entre la palabra y el texto más amplio del que pue-

res susceptibles de se présenter en position de matière sous chacune des formes généralisantes ou articulations de système qu'on a su momentanément se donner" (ibid., p. 24). No cabe duda de que no siempre se pueden explicar las prácticas lingüísticas de un mismo individuo refiriéndose a un solo tipo de sistematización de la lengua. Por el contrario, parece obvio que muchas veces - y probablemente en relación con la riqueza de su experiencia lingüística- el hablante elabora su habla a partir de una lengua tan polifacética, que resulta más fácil y quizás más conforme a la realidad representársela, más que como un todo sincrético armonioso, como una pluralidad de lenguas en competencia, poco distintas pero difíciles de superponer completamente. Aunque también el fenómeno escape otra vez al hablante medio, es frecuente observar que la elección de cada una de las variantes depende de la idea que se forme - exacta o equivocada- del tipo de situación enunciativa, puesto que no todos los "niveles de habla" se ajustan a cualquier circunstancia. Por dar un ejemplo concreto, detrás del doble comportamiento de los francohablantes frente al problema de la concordancia temporal (a lo antiguo, es decir aproximadamente tal como sigue practicándose en el castellano actual, o, más comúnmcntc, a lo moderno, descuidándola), se prefiere ver ahora la existencia de dos modelos en la lengua, uno heredado, otro, más reciente, innovado, alternativamente preferidos por el hablante que ha adquirido y conservado los dos (véase sobre el particular J. C. Chevalier, Verbe et phrase (Les problemes de la voix en espagnol et en français), Éditions Hispaniques, Paris, 1978, pp. 60-61). Pero, si bien permite efectivamente aislar para un mismo hablante, detrás de una capacidad lingüística, de cierto polimorfismo, varias facetas desiguales en algunos de sus elementos constitutivos, tal aspecto proteico no implica que se ajuste a pautas caprichosas. Por un lado, cada una de estas facetas conserva por su propia cuenta su cohesión interna, y, por otro lado, fijándose en una sola a la vez, el hablante evita fácilmente todo riesgo de incoherencia. 
den formar parte - se manifiesta bajo fisonomías muy diversas según quien hable, y según cuándo, dónde, por qué y a quién se hable.

Para designar esos dos tipos de intervención del hombre en el lenguaje, se han utilizado en la psicomecánica los dos términos de glosogénesis y de praxeogénesis ${ }^{15}$. Separadas por sus objetivos y sus resultados, las dos son, cada una a su modo, dependientes del tiempo del que se alimentan. Pero, si bien el tiempo glosogenético se confunde con el de la historia, no todo el tiempo praxeogenético se desarrolla según el mismo eje. Por supuesto, lo más a menudo, el hablante medio no tiene conciencia de tantas génesis por considerar ingenuamente, primero, que el lenguaje se reduce al habla, es decir a su movilización ${ }^{16}$, y segundo, por recibir probablemente el habla de los demás como un todo estático, más que como un auténtico proceso ${ }^{17}$.

\section{Rasgos distintivos de la lengua y del discurso}

A la lengua y al discurso corresponden el lenguaje potencial y el lenguaje efectivo, respectivamente. Las dos entidades en las que se divide el lenguaje como totalidad se oponen por varios rasgos. El discurso, concreto y material, es fácil y directamente observable, mientras que la lengua, abstracta e inmaterial, sólo se da a conocer por sus manifestaciones discursivas. Así, frente al primero, de carácter explícito obligado por contener exclusivamente unidades de expresión, la segunda, constante en la mente de cada individuo normal, no sale de lo implícito, por contener sólo unidades de representación ${ }^{18}$. Además, si bien el discurso, intermi-

15 VALIN, "Introduction", pp. 15 ss. Se ha modificado levemente la terminología (sustituyendo el sufijo - genie por el de -génesis) para armonizarla con otros términos de constitución parecida, los generalmente usados en psicomecánica con el segundo más bien que con el primero (no obstante, se encuentra el de idéogénie en MANTCHev, como se verá más adelante).

16 Sobre el carácter inconsciente de la lengua en el hablante sin educación gramatical y también su instantaneidad, véase VALIN (ibid., p. 31).

17 “[...] chacune des langues du passé est présentement le produit d'un procès de construction, que nous appelons gloso[genèse], procès en cours depuis des millénaires et des millénaires, le sujet parlant ayant, à tout moment, la faculté d'utiliser, à des fins momentanées et diverses, le résultat à ses yeux permanent - mais en réalité seulement durable- de ce procès multimillénaire de construction, ce qui constitue linguistiquement une autre forme d'activité à laquelle nous donnerons le nom de praxéo[genèse]'” (ibid., p. 15).

18 "Les representations [...] sont des unités construites et qui tirent de leur construction, des opérations successives qui la constituent, la totalité de leurs attitudes. Les expressions sont des phrases dont la signification tient partie aux 
tente y efímero, es de índole discontinua según se rompa o no el silencio, la lengua, duradera y persistente, tiene un carácter continuo. De modo que, mientras que en condiciones normales cada hablante controla su producción discursiva, no parece exagerado pensar que, por el contrario, nadie tiene una conciencia clara del fantástico haz de posibilidades de habla que lleva en sí, por lo menos desde el momento de la infancia en que, a partir de un entorno socializado, han logrado construirse por su propia cuenta los primeros atisbos de la lengua ${ }^{19}$.

\section{Relaciones entre la lengua y el discurso}

¿Qué tipo de relación tienen entre sî? Siendo la lengua un resultado adquirido, aunque constantemente puesto en tela de juicio, un resultado siempre a disposición de cada hablante de una misma comunidad, constituye para él un bien previo, lenta y paulatinamente elaborado con el tiempo por sus antepasados y, por eso, viene a constituir, a la manera de la parte sumergida de un iceberg, la pieza fundamental del lenguaje. La pieza que, transmitida de generación en generación, proporciona a todos la posibilidad de hablar en un idioma particular, eximiéndolos de tener que inventar o improvisar un lenguaje cada vez que les venga el deseo de expresarse por medios lingüísticos. Sabemos que a veces se da un discurso sin lenguaje, es decir sin lengua, a pesar de las apariencias: es lo que pasa cuando el papagayo logra repetir alguna que otra oración; sería también el caso, por ejemplo, si algún cantante occidental consiguiese reproducir y grabar la letra de una canción en japonés, sin conocer dicho idioma. Pero, no existe lenguaje sin lengua. Mientras subsista ésta, no desaparecerá el idioma correspondiente. Lógicamente, ningún idioma debería calificarse de muerto, antes de averiguar que no pervive su lengua en ningún hombre. De todos modos, los idiomas supuestamente muertos son muchas veces idiomas que han dejado de

représentations qu'elles articulent pour leur édification, partie aux circonstances où elles naissent"' (Chevalier, op. cit., p. 13).

19 ' Ce qui revient à dire que l'acte de langage s'insère entre deux diachronies dont l'une porte l'existence permanente et continue de la langue, et l'autre, l'existence momentanée et intermittente du discours. Ces deux diachronies dont la progression est infiniment lente, en comparaison de la rapidité avec laquelle s'efr fectue l'acte de langage, peuvent être, dans une analyse synchronique du langage, assimilées à des états de repos [...]"' (VALIN, Petite introduction..., p. 33, n. 3). 
hablarse, pero que podrían y pueden volver a usarse: en el caso clásico del latín, existen hoy en día métodos audio-orales que permiten a cualquiera aprenderlo como si siguiera siendo de uso corriente.

La lengua, piedra angular del lenguaje, proporciona, en su completa abstracción ${ }^{20}$, un conjunto sistematizado de representaciones. Pero en ningún caso se trata de entidades totalmente prefabricadas, inmediatamente dispuestas, sin adaptación, a servir para el discurso que las necesite. La psicomecánica las representa como programas abstractos de las operaciones mentales a las que debe lanzarse el hablante cuando quiere comunicar lo que le produce o lo que le sugiere la percepción de tal o cual porción del universo extralingüístico ${ }^{21}$. Tal concepto permite conciliar con cierta armonía el aspecto social y colectivo del lenguaje y la estabilidad relativa que le impone su funcionamiento, inscritos los dos en su faceta potencial, con la peculiaridad de cada acto lingüístico individual y también con la creatividad del hablante, el cual, en última instancia, es quien adopta y adapta, entre otras posibles, la explotación que mejor se ajuste al propósito de su discurso ${ }^{22}$. Y efectivamente, si a la psicomecánica se le reconocen algunos méritos, entre ellos parece lícito incluir probablemente los dos siguientes: $1^{\circ}$ ) gracias a su operatividad básica, el de destacar a la vez el aspecto programático y genético - por no decir generativo- del lenguaje; $2^{\circ}$ ) el de asentar la rigidez imperativa de su arquitectura -en sincronía y en el nivel individual- y, al mismo tiempo, de privilegiar sin contradicción la flexibilidad, ma-

${ }^{20}$ La previsión de materialización fónica que cada unidad de la lengua asocia a su significado potencial no es más que un patrón de configuración fonemática, constituida por una secuencia tácticamente ordenada de fonemas o unidades de segunda articulación, que proponen la representación de manifestaciones fonéticas o, por decirlo de otro modo, una clase de realizaciones fonéticas, es decir, finalmente, algo exclusivamente abstracto (la famosa "imagen acústica" de Saussure).

21 "'Ce système général de la langue habite la pensée sous la forme d'un savoir acquis empiriquement et grâce auquel est continûment offerte à la pensée la possibilité de se donner, au moment opportun, une représentation de tout ce qui est pour l'homme, dans un état donné de civilisation, matière d'expérience (interne ou externe), c'est-à-dire objet de pensèe"' (VALIN, Petite introduction..., p. 77).

${ }^{22}$ Es obvio que para muchos, quizás la mayoría, en vez de derivarse directamente del conjunto de sistemas contenidos en la lengua, no pocas veces es ante todo repetición de prácticas ajenas o de modelos normativos de cierto alcance. Cuando se da el caso, cuando dimite la creatividad del hablante, sin embargo se puede estimar que queda salvada su iniciativa, aunque se ha limitado a seleccionar modos de hablar elaborados por otros. 
ravillosa aunque limitada, de los usos a que se prestan las operaciones mentales que la constituyen. Como se ha recalcado varias veces $^{23}$, todo ello se debe al planteamiento y al método de investigación de la lingüística guillaumiana que, aunque no lo consiga siempre, procura reducir la multiplicidad de los valores discursivos de un mismo ente lingüístico, por muy alejados que parezcan -y aun cuando sus diferencias los hagan aparentemente contradictorios-, a una condición común que es la posición y el cometido de este ente dentro del sistema que lo contiene ${ }^{24}$.

Siendo así las cosas, la lengua tiene con el discurso una relación de anterioridad no sólo diacrónica, sino también sincrónica. Lo precede como realidad históricamente pre-existente, a la par que antecede evidentemente a los individuos de toda la comunidad. Pero, más aún: como lenguaje potencial, frente al efectivo, es la condición misma del discurso que, por consecuencia, de ella se deriva.

\section{Tránsito de la lengua al discurso}

El tiempo discursivo creador de la unidad del discurso.- Cuando se examina la transición de lo potencial a lo efectivo, y el tiempo que necesita, nos encontramos en realidad con dos especies de tiempo operativo. El primero, o tiempo discursivo, corresponde a una génesis cuyos instantes sucesivos se perciben con facilidad, que es la génesis de las unidades de que se compone el discurso, o sea las oraciones. Como lo enseña la experiencia lingüística más común - activa y pasiva-, hace falta cierta duración, cierta cantidad de tiempo para ordenarlas, una cantidad que, dado el carácter lineal del habla, es fácil de notar y de evaluar.

El tiempo glosológico creador dé la unidad de lengua. - El segundo tiempo, que se propuso llamar glosológico, es el empleado para efectuar las operaciones sistemáticas virtuales incluidas en los esquemas que encierra la lengua. En nuestros idiomas, nos permite realizar otra génesis, la de las unidades seleccionadas para la constitución de las oraciones, es decir los vocablos tales como los conocemos. De este tiempo, en cambio, no tenemos ninguna conciencia, probablemente porque no rebasa los límites de un solo

${ }^{23}$ Por ejemplo, Valin, Petite introduction..., p. 76.

24 "'Les valeurs diverses d'expression qu'un seul et même mot est susceptible de prendre momentanément dans le discours ne sont que les consequences, possibles et nécessaires de conditions posées en langue par une certaine représentation, laquelle est précisément le signifié de puissance dont le signe linguistique est le support"' (ibid., p. 81). 
instante, y porque sólo el pensamiento culto logra penetrar en la interioridad de tan corto lapso. Pero importa poco, pues su nulidad aparente o aproximada no impide que se analice como positivo.

El primero, el tiempo operativo discursivo, soporte de la construcción de la oración, al extenderse sobre varios instantes, se impone a la observación con la fuerza de lo evidente. La percepción del segundo, el glosológico, requiere mayor atención. Pero, en realidad, el postulado de su existencia procede de la simple proyección en el nivel potencial de lo encontrado en el nivel efectivo: el tiempo operativo glosológico no es más que la réplica del tiempo operativo discursivo, del que sólo se distingue por el tipo de unidad que permite edificar, por su considerable reducción, y por su carácter inconsciente ${ }^{25}$. Y si se reconoce que la oración viene elaborándose en el respeto de unas leyes combinatorias preestablecidas, no hay por qué negarse a admitir que el vocablo procede igualmente de una construcción previa, realizada en conformidad con unos programas mentales pre-definidos ${ }^{26}$.

\section{Esquema global del acto lingüistico}

Hablar, pues, es edificar discurso, y construir oraciones es partir de una potencialidad permanente para llegar a una efectividad intermitente. Si se quiere abarcar la totalidad del acto lingüístico, hay que cubrir todo el espacio que separa el campo de las representaciones del de las expresiones. Para dar idea de tal tránsito, la psicomecánica propone el esquema binario dinámico y adverso, utilizado como instrumento de explicación a fin de dar cuenta de los mecanismos mentales constitutivos de los sistemas lingüísticos, generalmente producidos por una oscilación entre lo general y lo particular.

Por consiguiente, entrar en un acto lingüístico es emprender un movimiento de actividad de tránsito entre dos puntos estáticos, y pasar de un estado de reposo inicial a otro estado de reposo final ${ }^{27}$.

${ }^{25}$ VALIN justifica la separación entre el tiempo discursivo y el glosológico, apoyándose en la distinción entre el macro-tiempo materializado por una duración perceptible y el micro-tiempo que escapa a toda percepción ("Introduction", pp. 34-35). Véase también Skrelina, art. cit., p. 87.

${ }^{26}$ VALIN, Petite introduction..., p. 47.

${ }^{27}$ Ibid., p. 32; véase supra, n. 19. 
Figura I

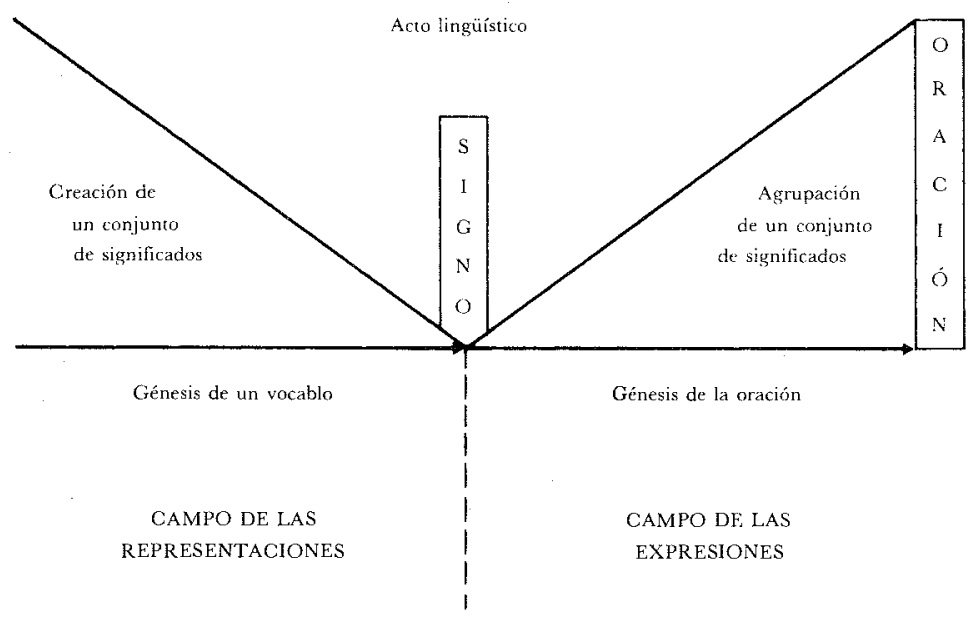

GÉNESIS MENTAL (LEXIGÉNESTS) DEL VOCABLO NOMINAL

\section{La verdadera unidad funcional del lenguaje}

El análisis lingüístico permite adentrarse en la palabra y dividirla en elementos de primera articulación dotados todos ellos de un significado particular, aunque no les corresponda siempre una configuración única ${ }^{28}$. Sin embargo, no cabe duda de que la arquitectura oracional no estriba en tales morfemas, sino en asociaciones de nivel operativo superior que los contienen: en nuestros idiomas, se trata de los vocablos, que son los primeros elementos viables y atónomos. Obviamente, ocurre que en la oración aparezcan morfemas aparentemente aislados; pero, en realidad, se trata sólo de palabras monofonemáticas en función de vocablos.

Está claro que, salvo para los vocablos sin ninguna posibili-

${ }^{28}$ Como se sabe, los elementos de primera articulación han sido llamados monemas por A. MARTINET que los divide en lexemas y morfemas (Elements de linguistique structurale, A. Colin, Paris, 1967, $\$ 1.9$ y 4.19). Seguimos aquí la terminología de PotTiER, por lo menos la de Linguistique générale, théorie et description, Klincksieck, Paris, 1974, $\$ 29,22,62$, y de Présentation de la linguistique. Fondements d'une théorie Klincksieck, Paris, 1967, p. 15, en vez de la de Systématique des éléments de relation, Klincksieck, Paris, 1962, pp. 107-109, 95 y 112 (véase además "Hacia una semántica moderna”, en Lingüística moderna y filologia hispánica, versión esp. de M. Blanco Álvarez, Gredos, Madrid, 1968, pp. 106-110, 2.12; y Grammaire de l'espagnol, P.U.F., Paris, 1969, p. 29). 
dad de flexión - o sea, invariables-, cada uno de ellos, antes de ser seleccionado para participar en la edificación de la oración, debe adaptarse al papel preciso que en ella se le reserve. Ello no impide que constituya la verdadera unidad de la lengua, la única forma aprovechable del lenguaje potencial para cualquier agrupación discursiva, desde el sintagma a la oración, unidad del lenguaje efectivo, pasando por la proposición ${ }^{29}$.

\section{La concepción psicomecánica}

Para describir el trayecto mental seguido en la construcción de la palabra, lo que llamaremos en adelante la lexigénesis, la psicomecánica propuso en un principio su habitual esquema binario y adverso. La tradición gramatical más antigua nos enseñó a separar, en cada palabra flexionada, un radical y una(s) desinencia(s). Para dar cuenta de cada uno de estos bloques en la organización interna del vocablo, se ha utilizado después la división entre lexemas y gramemas, que, como se ha recalcado con insistencia, subraya una diferencia más de función que de índole, y se cifra en la separación de conjuntos débil o fuertemente cerrados ${ }^{30}$.

Guiada por una orientación resueltamente operativa, la psicomecánica concibe la lexigénesis como un proceso dinámico, divisible en dos fases constitutivas, de las que sucesivamente se derivan dos especies de resultados. Se puede describir, pues, como una doble génesis: una génesis material, o ideogénesis, creadora de una sustancia semántica particular sin otro cometido que el propiamente nocional, y una génesis formal, o morfogénesis, creadora de una serie de sustancias, semánticas también, pero que sirven para aprehender el resultado de la ideogénesis. Simplificando, diremos que la primera produce la sustancia material del vocablo, y la segunda su sustancia formal, dado que, en nuestros idiomas, no se puede evocar ningún concepto sin tener que asociarlo, se quiera o no, a un aparato formal, sin el cual quedaría lingüísticamente inasequible e inservible por hallarse entonces privado de

${ }^{29}$ Lo ha recalcado precisamente MANTCHEV "Nous réfutons la thèse des écoles américaines suivant laquelle c'est le morphème [...] qui est l'unité minimale $[\ldots]$. Cette thèse relève d'une conception classificatoire dépassée du langage et non d'une conception opératoire. Une unité est un élément construit ou ce n'est pas une unité, c'est un élément constitutif, une composante, intégrée dans l'unité' (La genèse..., p. 115, n. 13).

30 Véanse otra vez Martinet, op. cit., $\$ 4.19$, y Pottier, Systématique..., p. 95 y Linguistique..., $\$ 62$. 
toda posibilidad de combinación ${ }^{31}$. La figura II corresponde al modelo de lexigénesis común a la mayor parte de los psicomecánicos, porque es el que describió primero Guillaume.

Figura II

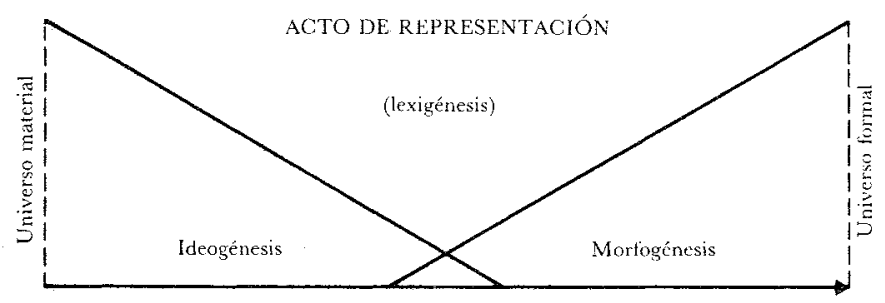

La imbricación de sus dos fases.- Pero ya Guillaume había hecho énfasis repetidas veces en la imbricación de las dos operaciones. En efecto había puntualizado que, si bien no se puede imaginar ninguna aprehensión sin disponer previamente de algo que aprehender, en cambio, la morfogénesis puede iniciarse antes de que finalice la ideogénesis, produciéndose así la superposición parcial de la primera por la segunda. Así quedaba asentada la posible coexistencia dentro del desarrollo lexigenético de sus dos procesos constitutivos; y, para la morfogénesis, la facultad de anticiparse, con respecto a la ideogénesis, es decir la capacidad de arrancar a distancia muy reducida del principio de la ideogénesis, siempre que esa distancia sea superior a cero. Además, aplicando esa precocidad fue como Guillaume pudo explicar la desaparición progresiva de la declinación nominal en la mayor parte de los idiomas neolatinos por el comienzo cada vez más anticipado de la segunda fase ${ }^{32}$. Pero, fuera de esa tentativa de interpre-

31 ' Le problème grammatical se pose toutes les fois qu'un propos limité entre comme unité composante dans le système d'un propos plus vaste. Il y a problème grammatical quand une phrase entre comme unité composante dans le système d'une phrase plus étendue [...]. Et il y a de même problème grammatical [...] quand le mot entre comme unité composante dans la phrase. Dans l'un et l'autre cas on voit le composant faire appel, afin d'acquérir la convenance voulue, au composé plus large dont il devient une partie, à des déterminants qui ne lui appartiennent pas en propre, peuvent s'appliquer à d'autres composants, valent pour toute une classe, toute une espèce, et accusent par là une généralité relative d'autant plus grande que le champ de leur application est plus vaste, moins déterminé" (Langage..., p. 108).

32 "Esquisse d'une théorie psychologique de la déclinaison" (ibid., p. 102). 
tación, los esquemas que proponen sus escritos publicados no parecen tener en cuenta tal penetración.

\section{Una extrapolación personal del modelo}

Detrás de la lexigénesis, Guillaume había descubierto las dos operaciones mentales fundamentales subyacentes a su esquema predilecto: una particularizadora (llamada por él de discernement), y otra generalizadora (llamada d'entendement), es decir, respectivamente orientadas hacia la estrechez y hacia la anchura máximas. A cada una, y en este orden, corresponde cada una de las dos génesis. Sin embargo, profundizando más, había descubierto también que en cada una se repite la oscilación de lo general a lo particular y de lo particular a lo general ${ }^{33}$. Lo que conduce finalmente a representarse el trayecto lexigenético bajo una forma doblemente binaria, por repetición del mecanismo que estructura su división dentro de cada una de sus dos fases separables. Tal modelo, aunque con alguna dificultad y cierta vacilación en la descripción de Guillaume, tendía a asentar una simetría dinámica ${ }^{34}$.

En la figura III, hemos tratado de hacer más visible esta homología, y, para facilitar la percepción de las relaciones ideomorfogenéticas, hemos separado en dos planos horizontales disparejos (planos I y M) las dos fases sucesivas. Como se ve, en cada una de ellas se distinguen dos momentos, según esté o no en coincidencia temporal con parte de la otra.

La ideogénesis. - La ideogénesis se puede partir en premorfogenética y co-morfogenética: $\mathrm{Ui}_{o} \rightarrow \mathrm{U}^{\prime} \mathrm{i}_{x}$ representa lo que de ella se ha desarrollado, antes de que naciera la morfogénesis. Avanzando más, desde $\mathrm{U}^{\prime} i_{\boldsymbol{x}}$ hasta $\mathrm{Ui}_{\boldsymbol{n}}$, se prosigue la primera, pero ya ha empezado la segunda. Mutatis mutandis, e invirtiéndose las cosas, del mismo modo se separan en el plano $\mathbf{M}$, $\mathrm{Um}_{o} \rightarrow \mathrm{Sm}_{n}$, co-ideogenético, $\mathrm{Sm}_{n} \rightarrow \mathrm{U}^{\prime} \mathrm{m}_{n}$ exclusivamente postmorfogenético.

Adentrándonos más en el mecanismo, encontramos las siguientes etapas. Lo que se recoge en $\mathrm{Si}_{n}$ es una noción peculiar. Ya es de índole distinta a la del universo extralingüístico de que se ha entresacado, puesto que su existencia se debe tanto o más a

${ }^{33}$ En Sistemática... (p. 34), MolHo parece haberse atenido a una presentación simplificada.

${ }^{34}$ Sobre la cuestión, remito a un trabajo personal, actualmente en preparación, que versará sobre la presentación psicomecánica de la lexigénesis a través de un conjunto de obras publicadas (del propio GuIllaume y de algunos de los que adoptaron total o parcialmente sus principios lingüísticos). 
Figura III

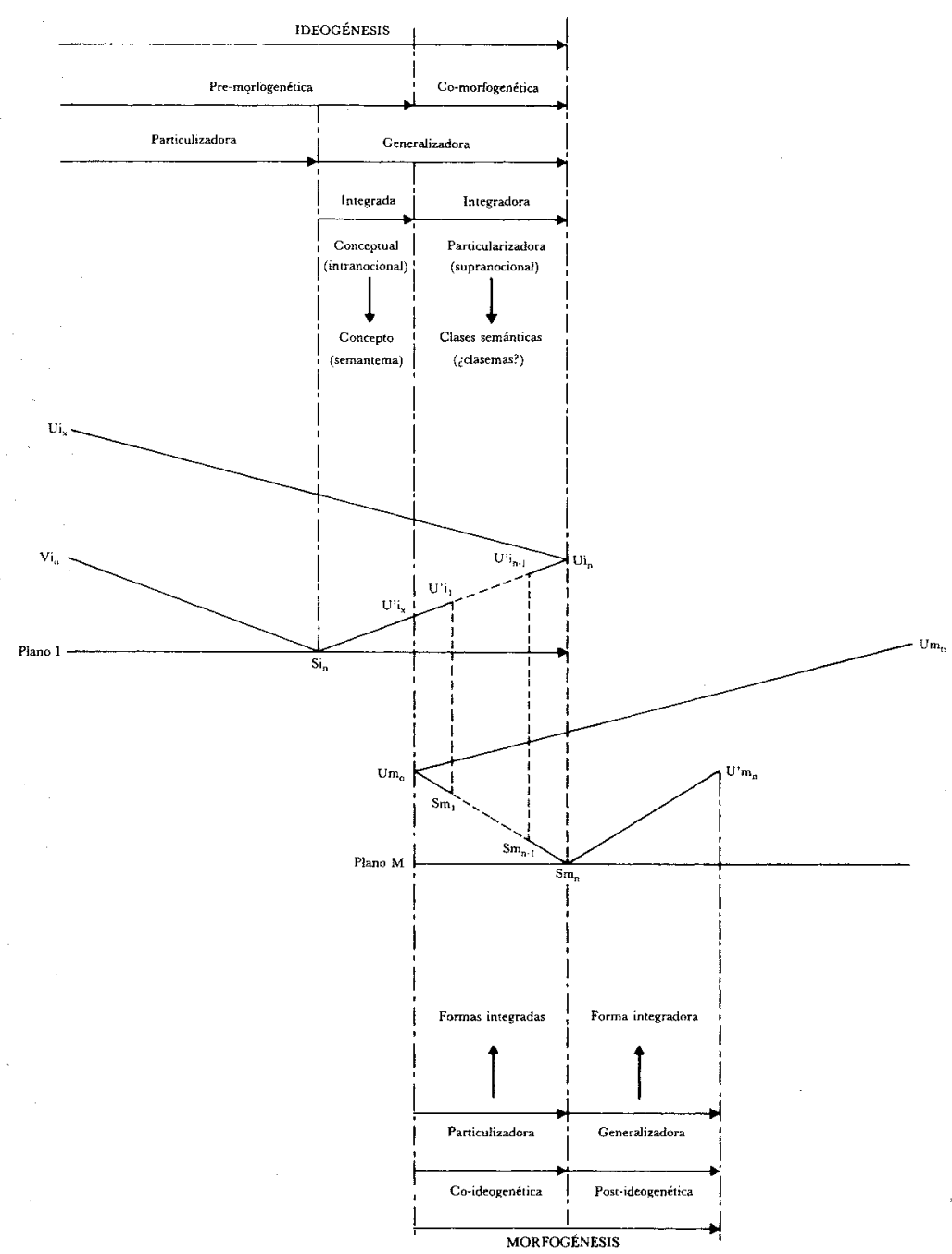

U'i $\mathbf{i}_{x}=$ universo extralingüístico de partida.

(ilimitadamente divisible: $x$ es una serie abierta de entes todavía sin posible aprehensión lingüística)

$\mathrm{Um}_{n}=$ universo lingüístico de llegada

(limitadamente dividido: $n$ es una serie cerrada de entes aprovechables para la aprehensión lingüística del universo extralingüístico)

$\mathrm{Ui}_{\mathrm{o}}=$ universo ideogenético de partida

$\mathrm{Si}_{n}=$ lo más particular de la ideogénesis

$\mathrm{Ui}_{n}=$ lo más general de la ideogénesis

$\mathrm{Um}_{o}=$ universo morfogenético de partida

$\mathrm{Sm}_{n}=$ lo más particular de la morfogénesis

$\mathrm{U}^{\prime} \mathrm{m}_{n}=$ lo más general de la morfogénesis 
su percepción que a su realidad. Sin embargo conserva con alguno de sus fragmentos, o mejor dicho, con la especial experimentación que ha conducido a aislarlo, una relación estrecha. Un vínculo que, progresivamente, va a ir debilitándose, conforme la noción obtenida se generalice, hasta llegar a constituir un auténtico concepto, con el cual, sin salir de la noción considerada, se obtiene un primer grado de generalización.

A partir de ahí, el concepto, entrenado en el mismo movimiento, se ve integrado, desde $\mathrm{U}^{\prime} \mathrm{i}_{x}$ hasta $\mathrm{Ui}_{n}$, en unas capas semánticas de extensión mayor, también presentes en la ideogénesis de otros muchos vocablos parecidos. La adquisición de estos rasgos, que parecen tener cierto parentesco con los clasemas de Pottier ${ }^{35}$, ya encamina la palabra en construcción a cierto tipo de formalización, y por eso merecen el calificativo de preformalizadores. Como veremos en el caso concreto del sustantivo, por su aportación como por su ubicación relativa, aunque ideogenéticos, presentan ciertos caracteres que los aproximan a las formas preconclusivas.

La morfogénesis. - Pero, como hemos dicho, conjuntamente, a plomo con U'i $i_{x}$, ha arrancado la morfogénesis. Lo que proporciona primero es cierto conjunto de formas integradas sucesivas que, progresivamente, van a conducir hacia cierto tipo de forma general, la única compatible con esta particularización morfogenética. Esta última no proviene de la índole separada de cada una de las formas preconclusivas que, como formas, no dejan de

${ }^{35}$ Se trata de elementos por encima o más allá de los semas denotativos específicos (constitutivos del semantema), y al lado de los semas connotativos virtuales (los cuales definen el virtuema), a cotejar con los semas denotativos genéricos (cuya agrupación forma el clasema). Pero se trata de entes sémicos de una abstracción aún más extremada que la de los semas genéricos, como incita a pensarlo en especial su resistencia a una discriminación: la que, aplicada a la vez a los semas denotativos específicos y genéricos, condujo a POT. TIER a dividirlos en descriptivos / aplicativos. A pesar de todo, algunos de los clasemas parecen tener un grado de generalización comparable, entre ellos por ejemplo el de discontinuidad que POTTIER aísla en el semema de armoire (Linguistique. . ., p. $70, \S 64$ ). Al fin y al cabo, puede que, de los semas genéricos a tales elementos, la diferencia sea sólo de grado. Y tal vez, para definir éstos a partir de aquéllos, sería aceptable decir que no se trata de todos los semas genéricos, sino, exclusivamente, entre ellos, de los que repercuten en el plano morfogenético de la lexigénesis. De ahí se sigue, como bien podría imaginarse, que sólo es posible identificarlos y aislarlos dentro del marco de cada idioma; incluso si el parentesco histórico o un parecido estructural provoca el encuentro de una misma categoría entre los idiomas de un grupo o en un conjunto de idiomas separados. 
poseer un grado importante de generalidad, sino de su asociación: porque tal agrupación es la que finalmente determina un solo tipo de forma final, aun más general que las integradas de que procede. La forma conclusiva se conoce como la forma del vocablo, tradicionalmente denominada parte del discurso, pero que valdría más llamar, porque lo es, parte de lengua.

Como acabamos de sugerir, a pesar de la aparente disponibilidad de las formas gramaticales para cualquier contenido, y también a pesar de la aparente compatibilidad de los significados con cualquier forma - dejando de lado el peso de las eventuales presiones normativas-, tal posibilidad y tal compatibilidad se supeditan a un mínimo de coherencia entre los atributos postconceptuales y las formas preconclusivas. Es decir que la morfogénesis particularizadora será lo que permita que sea la ideogénesis (generalizadora) supranocional ${ }^{36}$.

\section{Aplicación a la lexigenésis nominal románica}

Descripción somera de su ideogénesis. - Para concretar un poco las cosas, presentamos en seguida las relaciones ideomorfogenéticas tales como las describió Moignet para el sustantivo francés, con la convicción de que pueden aplicarse sin violencia al caso del español ${ }^{37}$. En la lexigénesis nominal, en la porción $\mathrm{U}^{\prime} \mathrm{i}_{x} \rightarrow \mathrm{Ui}_{n}=\mathrm{Um}_{o} \rightarrow \mathrm{Sm}_{n}$ en la que coexisten las dos fases, ha distinguido las cuatro capas sucesivas siguientes: 1 . la clase, 2. el morfismo, 3. la potencia, y 4 . lo que se podría denominar la determinación ontológica. En cada uno de los cuatro puntos se abre una alternativa, a partir de la cual se van a definir los rasgos ideogenéticos supranocionales ( $=$ post-conceptuales) y morfogenéticos integrados ( $=$ preconclusivos) relevantes. En 1. la ideogénesis puede vacilar entre sumir o no la noción previamente discriminada y conceptualizada en el conjunto de lo animado ( $+\mathrm{An} /-\mathrm{An})$, $y$, consecuentemente, la morfogénesis se adapta haciendo corres-

${ }^{36}$ Esta concepción viene a coincidir con la que manifestó SKRELINA cuando dijo, apoyándose en Guillaume: "Les déterminants en question [du vocable] sont des catégories verbales ou nominales qui s'étaient formées au cours de la grammaticalisation [ = morphogenèse] des mots. Le procédé est lié, selon Guillaume, à l'universalisation de la vision du monde. La face double de ce monde, présentée en union et en opposition de l'space et du temps, trouve sa réplique dans la distinction grammaticale du nom et du verbe" (art. cit., p. 88).

${ }^{37}$ G. MoIGNET, Systématique de la langue française, Klincksieck, Paris, 1981, $\$ 40$ y 54 . 
ponder un género gramatical sea alternante (Mase / Fem), sea sin alternancia (Mase / $\phi$, o Fem / $\phi$ ). En el punto 2. el morfismo propone integrarla en lo continuo o en lo discontinuo, y en el plano $M$, según el caso, hay que conformarse, desde el punto de vista numérico, con un singular obligado - eventualmente un plural interno por falta de toda posibilidad de multiplicación- o quedan abiertos el singular y el plural externo oponibles — también queda posible el plural interno. Por lo que atañe al tercero, punto en que se prevé el papel sintáctico de la palabra en el conjunto oracional, evaluando entre un máximo y un mínimo de potencia, no tiene gran relevancia en el estado actual del francés o del español, dado que no repercute en la morfología interna del sustantivo; sin embargo, como es fácil observar, influye sobre su formalización externa, y en particular, en cierta medida por lo menos, sobre su dependencia o su independencia de todo predeterminante (las distintas clasès de artículo, entendido en sentido amplio) ${ }^{38}$. Finalmente, con el 4. llegamos al último momento de la ideogénesis y casi de la lexigénesis: según lo que se necesite constituir como unidad de lengua, encaja o no el concepto en la clase de los entes; entonces es cuando se le reconoce o no el estatuto ontológico. Hasta aquí se ha venido enriqueciendo por la adición de unos rasgos no específicos que han incrementado constantemente su carácter general, tanto por el lado ideogenético como por el morfogenético, es decir, al fin y al cabo: su facilidad de empleo, su disponibilidad, su posibilidad de combinación. Ahora, una de dos. O se le atribuye la cualidad de un auténtico ente, y entonces lo que precisamente denota sólo lo puede referir a sí mismo: su aportación material sólo la destina a su consumo propio, porque lleva en sí la capacidad de soporte. Por eso, posee la persona (cardinal) $o$, en términos psicomecánicos, la incidencia interna ( = auto-incidencia) que lo hace autoportado(r) e independiente en algunas circunstancias - además, desiguales entre el francés y el español, como lo muestran las posibilidades de encuentro del artículo cero en uno y otro idioma. $O$ se le concibe a la inversa,

38 Véase infra, pp. 44-47. De todos modos, la sola práctica de los dos idiomas español y francés permite darse cuenta de que lo que la psicomecánica llamó, a propósito del segundo, el caso sináptico del nombre sustantivo, no presenta, en el primero, la misma capacidad. Como se sabe, la diferencia se manifiesta en la función de complemento primario. Sobre el particular, además de una alusión directa del mismo Guillaume, en Leçons..., t. 2, pp. 171-172 y 195, remitimos a dos artículos de MolHo, "La question de l'objet en espagnol", $V R, 17$ (1958), 209-219, y “La grammaire de l'objet en espagnol", TLL, 18 (1980), 213-225. 
y entonces su aportación material sólo podrá insertarse en el contenido de otra parte de lengua capaz de proporcionarle el soporte de que carece: se tratará de un elemento nominal por supuesto, pero de un adjetivo, aloportado y dependiente, de incidencia externa (de primer grado).

Coherencia interna de la lexigenésis. - Como se transparenta en las vacilaciones del propio Guillaume en sus dos versiones de la lexigénesis ${ }^{39}$, parece haber quedado parcialmente pendiente el problema de la bipartición del universo formal $\mathrm{Um}_{n}$ en el que acaba por recaer toda la lexigénesis. $\mathrm{Si}$, como realmente llevan a pensar nuestros idiomas, todo el lenguaje se reparte en dos campos distintos, espacial y temporal, algunas de las sugerencias más o menos furtivas de Moignet ${ }^{40}$ inducen a pensar que la postura frente a esta alternativa ya se define en los primeros momentos de la lexigénesis. Por consiguiente, parece posible admitir que el porvenir morfogenético de cada vocablo se juega desde los inicios de la ideogénesis. De hecho, la posible modificación de la orientación primitiva (espacial o temporal) de algunas palabras, a la que se deben los verbos denominales y los nombres deverbales ${ }^{41}$, facilitada por ciertos infijos de derivación, tendería a acreditar la existencia previa de tal orientación. De modo que la índole de la forma final se supedita a una opción inicial nocional o prenocional.

Evidentemente, todos los rasgos supranocionales por fuerza tienen que casar con la primera imagen que la mente se haya formado de la noción en vías de definición, sea como la de un ente - fuera de toda manifestación en el tiempo-, sea como la de un fenómeno - independiente de tal o cual ente particular, aunque indisociable de un ser arquetípico. Así que, progresivamente, esa opción inicial es lo que determina toda la formalización del vocablo, si no sus especificaciones precisas, por lo menos las distintas alternativas en las que tiene que especificarse: en total, el conjunto formal, y en particular la índole nominal o temporal de la parte de lengua, no son sino el eco tardío de una opción ideogenética temprana sin modificar.

${ }^{39}$ Las dos versiones mencionadas se hallan respectivamente en "Discernement et entendement dans les langues mot et partie du discours" y "Esquisse d'une théorie psychologique de la déclinaison", ambos artículos de 1939, reimpresos en Langage..., pp. 87-107.

${ }^{40}$ Systematique..., $\$ 21,39,69,41-42,54,71$ y 338.

41 Véase Moignet, ibid., $\$ \$ 39$ y 46, y Molho, Sistemática. . ., pp. 37-39. 
De los significados a los significantes eventuales

Si dejamos el plano de los significados - gramaticalizados o no: de los gramemas o de los lexemas- por el de los significantes (ateniéndonos a la terminología estrictamente saussuriana), se dan dos tipos de situación. Algunos vienen asociados con configuraciones fónicas particulares: en el caso del sustantivo, se trata de los estratos que definen la clase y el género, el morfismo y el número. Aunque no se observe siempre, puede que cada una de las opciones correspondientes se exprese por una semiología transparente. En cambio, ni la forma conclusiva, ni la primera elaboración ideogenética tienen con qué manifestarse. La elección que a su propósito se ha hecho, sólo se descubre, indirectamente, a partir de las otras elecciones -ideogenéticas y morfogenéticasoperativamente posteriores, reflejadas por sus significantes propios.

Huelga decir que, de todos modos, queda una disimetría importante entre las dos fases. En efecto, sólo en la morfogénesis se observa, aunque parcialmente, una correlación significado-significante. En la ideogénesis, la semiología globalmente asociada al producto conclusivo de la ideogénesis (recogido en $\mathrm{Ui}_{n}$ ) no transparenta ninguno de sus ingredientes. Esa realidad, puede que haya obstaculizado los progresos de la semántica no gramatical.

\section{Efectos graduales de la subducción}

Así no parece demasiado arriesgado asentar la existencia de un condicionamiento de la morfogénesis por la ideogénesis. Por si fuera necesario aún, la psicomecánica puede aducir una prueba, valiéndose de su teoría de la subducción, presentada y aplicada por Guillaume sobre todo en el campo verbal ${ }^{42}$. Recordemos que se ha denominado así al fenómeno de desmaterialización que, generalizando, a priori puede afectar a todo tipo de palabra. Si, después de Moignet, se considera la predicatividad como la designación de los datos de la experiencia ${ }^{43}$, se sabe que ésta varía

${ }^{42}$ Véase en particular: "Théorie des auxiliaires et examen de faits connexes" (1938), Langage..., pp. 73-86.

${ }^{43}$ Systematique..., p. 121, $\$ 176$ (y también, $\$ \$ 18-19$ ), y "L'opposition non / ne en ancien français", TLL, 7 (1965), 41-42. "Les parties de langue prédicatives ont pour fonction d'assigner une matière notionelle à une forme linguistique. Cette matière, élaborée en lexigenèse [= idéogenèse], est fournie par la conceptualisation des données de l'expérience humaine. Ce qui en fait la substance, c'est l'univers auquel la pensée humaine est afrontée, c'est tout ce que le hors-moi présente à la concevabilité du moi pen- 
mucho, no sólo de un tipo de vocablo a otro, sino también en una misma palabra, según la explotación que de ella se haga. A veces la disminución de tal predicatividad no repercute en la morfogénesis. Aunque se hable entonces, como se suele hacer, de "palabras gramaticales" -es decir sin sustancia material de tipo denotativo- fuera de leves modificaciones, su morfología interna tiene una fisonomía muy parecida a la de sus correspondientes predicativos. Por ejemplo, los pronombres, los artículos, ciertos adverbios, los auxiliares y asimilados - llamados por Moignet transustantivos, transadjetivos, transadverbios y transverbos ${ }^{44}$ - se asemejan mucho a los sustantivos, adjetivos, etc.

Pero, otras veces, la predicatividad se ha reducido tanto que acaba por afectar a la morfogénesis. Las preposiciones y conjun-

sant. Les parties de langue non-prédicatives ne sont pas, elles, adossées à l'expérience du hors-moi. Leur matière notionnelle, tout entière tirée du moi pensant, n'est faite que de ce que la pensée a pu saisir des conditions de son propre fonctionnement" (MolGNET, Systématique. . ., \$ 19). "[ . . .] nous avons défini les parties de langue non-prédicatives par leur contenu notionnel d'un haut degré d'abstraction, sans affinité, au moins directe, avec l'expérience de l'univers, et fondé sur une saisie opérée par la pensée de ses propres conditions de fonctionnement. Autrement dit, la lexigenèse des termes en cause est faite de formes en position de matière"' $(\$ 176)$. Fácilmente se observará, de una a otra de las dos citas, una leve modificación de la definición de la nopredicatividad. En efecto, mientras se da primero como procedente "du seul moi pensant", sin relación con su experiencia, después se le concede a pesar de todo cierta vinculación -indirecta - con tal experiencia. Se hallan otras huellas del segundo punto de vista, por ejemplo en el $\$ 29$ ("Ce que les parties non-prédicatives ont en commun, c'est leur absence de référence, au moins relativement directe, à l'expérience acquise par la pensée humaine dans son appréhension de l'univers extérieur'). Y efectivamente, parece preferible considerar la no-predicatividad como un caso particular de predicatividad menguada, aminorada. El caso en que, sin necesidad de rechazar toda relación con el no-yo - además difícil de negar - un vocablo ha depurado su contenido ideogenético hasta el punto de perder en poder denotativo lo que gana en posibilidades gramaticales. En resumidas cuentas, cada parte de lengua a punto de perder (una porción variable de) su predicatividad se halla capacitada, por el tratamiento reservado a su ideogénesis, más para formalizar partes más denotativas que ella que no para servir de vocablo preferentemente denotativo; valiéndose de otros términos de MOIGNET, "quitter la prédication", es "substituer une forme de pensée à la dénomination d'une sémantèse" (id.). V'áase también Pottier, Systématique..., pp. 107-112, y Lingüistica..., pp. 93-95, \$\$ 2-4.

${ }^{44}$ Systématique..., $\$ 177-179 . "$ “[...] on est $[\ldots]$ dans le domaine de la transcendance notionnelle, exclusivement. L'idéogenèse des termes en cause n'est pas fondée, au moins directement, sur les données de l'expérience, mais la morphogenèse est, à peu de chose près, de même nature que dans les parties de langue prédicatives qui se situent en vis-à-vis"' ( $\$ 181)$. 
ciones tienen indudable originalidad en comparación con las demás partes de lengua: esencialmente, su comportamiento especial se debe a una doble incidencia (con necesidad de dos soportes, en vez de uno, como mínimo $)^{45}$.

Es inútil repetir lo dicho por Guillaume sobre el proceso de auxiliarización de ciertos verbos, que interesa aquí porque ha mostrado cómo la depuración material acarrea repercusiones sintácticas. Me limitaré a recordar, como lo manifiestan algunos estados antiguos del futuro español (en especial el llamado futuro analítico) y el actual futuro portugués en el caso del verbo con complemento pronominal, que el grado de autonomía y de viabilidad del verbo haber o aver como palabra se ha ido reduciendo conforme crecía la importancia de su desmaterialización ${ }^{46}$.

Para volver al sustantivo, esa misma relación se echa de ver cuando, empleado sin artículo, viene detrás de ser o en posición apositiva (Juan es profesor, profesor en Valencia, se hizo célebre por sus novelas). En efecto, aquí, asistimos a una verdadera desustantivación formal, por la pérdida de la persona cardinal, porque ya no se trata de un elemento lingüístico autoportado(r). Corresponde a una alteración ideogénetica que lo priva de la posibilidad de denotar a un ser humano de cierta función, permitiéndole tan sólo referirse a la función, exclusivamente.

La psicomecánica suele definir el aligeramiento semántico debido a la subducción como el efecto de la intervención anticipada de la morfogénesis sobre una ideogénesis sin acabar, es decir que no ha alcanzado el término de su posible desarrollo ${ }^{47}$. La representación de la lexigénesis que hemos defendido hasta aquí conduce a modificar levemente tal definición. Porque, realmente, lo que parece anticiparse no es sólo la morfogénesis en su totalidad, sino, antes y además de ella, la ideogénesis co-morfogenética inclusive. Dicho de otra forma, la subducción de un vocablo, y en

45 " [... ] la transcendance est, non seulement notionnelle, mais aussi formelle, et ce qui est transcendé, c'est le système qui s'achève avec l'adverbe et se définit par l'incidence telle qu'elle fonctionne dans le système des parties de langue prédicatives" (ibid. \$182). Sobre el caso del verbo en general y del verbo transitivo sin transitivizar en particular, véase CHEvalier, op. cit., pp. $15-28$ y $98-107$.

${ }^{46}$ Tales diferencias han llevado a Guillaume a distinguir entre dos tipos de subducción interna (esotérica): inmanente cuando no altera la viabilidad y autonomía de la palabra (habla entonces de un elemento "stématique"), trascendente cuando se la quita (convirtiéndola en "astématique") (Langage..., p. 75).

47 Véanse Molmo, Sistemática..., pp. 132-139, y 185, y Molgnet, Systématique..., \$189-191. 
particular la subducción del vocablo nominal al que nos hemos limitado aquí, consiste finalmente en una conceptualización precipitada de su contenido semántico. Una conceptualización precipitada que, según su potencia, disminuye más o menos la peculiaridad nocional a la que podía pretender, capacitándolo para empleos más gramaticalizados, a veces asociados a alteraciones morfogenéticas únicamente perceptibles en el plano de su posibilidad de combinación (morfología externa).

\section{Conclusión}

El modelo modificado que hemos tratado de presentar para la lexigénesis quizás habrá que adaptarlo para que sea igualmente aplicable a todos los tipos de vocablos, y en particular al verbo. Es un trabajo que ya tenemos previsto. De momento, y en cuanto al verbo, lo que por lo menos se puede imaginar son los dos puntos siguientes: $1^{\circ}$ ) dada la simple índole verbal, las capas ideogenéticas con papel preformalizador resultan de cierta apreciación, no del mero contenido conceptual de la palabra, sino a la vez de la relación que la vincula a su soporte obligado - tradicionalmente llamado el sujeto-, y de la posición del conjunto verbo - soporte respecto de las distintas representaciones que el idioma proponga del tiempo (es decir todas las posibilidades de la conjugación); $2^{\circ}$ ) entre el francés y el español, por fuerza habrá que establecer diferencias más importantes que en el caso del nombre, porque, como se sabe, sólo el verbo castellano es capaz de autonomía sintáctica, sin tener que acudir a un soporte exterior.

Francis TOLLIS

Université de Pau et des Pays de l'Adour 